


\title{
Modelo de ruteo de vehículos capacitado y multiproducto aplicado a una empresa distribuidora de materiales para la construcción en Sincelejo, Sucre
}

\begin{abstract}
Fernando Morales Boteroํㅜㄹ Harold Martínez García², Jesús Sierra Manrique ${ }^{3}$, Gustavo Vergara Hoyos ${ }^{4}$
\end{abstract}

\section{Resumen}

En la actualidad, existen diversos factores que inciden negativamente en la competitividad y equilibrio de las empresas, uno de estos se debe a problemáticas de planificación de estrategias que ayuden a reducir los costos de operación. En el siguiente estudio se plantea una herramienta para la planificación del sistema de distribución de productos, la cual fue aplicada en una empresa dedicada al despacho al por mayor y al detal de productos para la construcción, ubicada en la ciudad de Sincelejo. Se construyó un modelo de programación entera mixta de ruteo de vehículos (VRP) que considera variantes de multiproducto, capacitado con flota heterogénea, con el cual se logró obtener mejoras en el proceso de asignación de rutas de la empresa, mejorando la eficiencia del sistema a través del aprovechamiento de la capacidad por envío en cada vehículo; además, se estructuró una nueva organización de tiempos de pedidos. De esta manera, se logró un aumento del 8.22\% del beneficio sobre el proceso de distribución.

1 Ingeniero Industrial. Corporación Universitaria del Caribe-CECAR. Correo: fernando.moralesb@cecar.edu.co; Orcid: https://orcid.org/0000-0002-7331-4567 2 Ingeniero Industrial. Corporación Universitaria del Caribe-CECAR. Correo: harold.garciam@cecar.edu.co; Orcid: https://orcid.org/0000-0001-8630-1450 3 Ingeniero Industrial. Corporación Universitaria del Caribe-CECAR. Correo: jesus.sierram@cecar.edu.co; Orcid: https://orcid.org/0000-0003-0483-7687 4 Ingeniero Industrial. Corporación Universitaria del Caribe-CECAR. Correo: gustavo.vergara@cecar.edu.co; Orcid: https://orcid.org/0000-0001-9034-7208 
Palabras clave: sistemas de distribución, ruteo de vehículos, modelo de optimización, CVRP, GAMS.

\section{Multi-product capacitated vehicle routing model applied to a construction materials distribution company in Sincelejo, Sucre}

Abstract

At present, there are several factors that negatively affect the competitiveness and balance of companies, one of which is oriented to planning problems of strategies that help reduce operating costs. The following study proposes a tool aimed at contributing to the planning of the product distribution system, which was applied in a company dedicated to the wholesale and retail sale of products for construction, located in the city of Sincelejo, The tool emphasizes in a model of optimization of routing vehicles (CVRP) subject to exact methods of mixed linear programming with binary variables, by means of which it was possible to propose improvements in the methods of assigning routes to each of these by a number determined of orders, including multi-products and multi-customers, improving the efficiency of the system through the use of the capacity per shipment in each vehicle, highlighting that in the research orders were taken for time windows established, in addition, the proposed model consider reference nodes distributed throughout the city

Keywords: distribution systems, vehicle routing, optimization model, CVRP, gams. 


\section{Introducción}

El transporte integra un factor relevante en la cadena de suministro, todos los días las organizaciones enfrentan el reto de planificar sus distribuciones de forma eficiente, garantizando la capacidad de sus vehículos para así cumplir con la demanda y la particularidad específica de cada orden. En la investigación de (Integrado et al. 2013) afirman que la complejidad de la distribución de productos, desde ciertos centros de acopio a sus clientes finales, contribuye en la gestión de algunos sistemas logísticos. Por lo tanto, el considerar una planificación adecuada puede llevar a un cuantioso ahorro, reflejado en la proyección de la empresa, dado que se estima que los costes del transporte figuran entre el 10\% y el 20\% del costo final de los productos.

El problema de ruteo de vehículos (VRP) puntualiza en la actividad de conseguir soluciones cercanas a la optimización y reducción de los costos de operación, también permite maximizar el nivel de clientes atendidos (Bermeo Muñoz and Calderón Sotero 2009). Además de ser un tema de alta relevancia, facilita el desarrollo de la planificación y regulación de sus componentes.

En la actualidad, aunque estemos inmersos en niveles de tecnificación, la mayoría de las organizaciones con operaciones de distribución y despachos continúan con el planteamiento de diseñar rutas de manera empírica, obviando herramientas de apoyo que los orienten a la mejora continua (González Vargas and González Aristizábal 2006). Un ejemplo claro es el caso de la PYME comercializadora y productora, ubicada en la ciudad de Sincelejo, Sucre. Esta es una empresa que fabrica y distribuye productos no metálicos para la construcción, además posee una flota de vehículos con diferentes capacidades, utilizados para el transporte de sus productos en todo el municipio.

En síntesis, la importancia de realizar una planificación de las rutas para la entrega del producto, se enfoca en la organización de la flota de vehículos para abastecer los pedidos o demanda pronosticada fundamentalmente, generando un valor agregado al eslabón de la distribución (Tokito 2018) Por lo cual, la siguiente investigación busca determinar las rutas, tipo de vehículos y cantidades de producto a enviar en la PYME de estudio, 
logrando programar los envíos de los pedidos con un tiempo de captura, con el fin de optimizar los recursos de la empresa.

\section{Marco teórico}

La investigación realizada parte de definiciones del área involucrada, a las cuales se hace referencia con la intención de contextualizar.

\section{Cadena logística de transporte}

Es un componente de la gestión de la cadena de suministro que gestiona, planifica e inspecciona los procesos de aprovisionamiento, producción y distribución, y tiene como finalidad la satisfacción del consumidor final (Lama and Genaro 2013).

\section{Problema de enrutamiento de vehículos (VRP)}

Está centrado en un problema de optimización combinatoria que conlleva a un análisis surgido a partir de distribuciones reales y aplicaciones logísticas de enrutamiento, integrado por los siguientes componentes: la red de carreteras, los depósitos, los clientes, los vehículos y los conductores (Cornejo Sánchez and Puente 2005).

\section{Problema de ruteo de vehículo con capacidad limitada (CVRP)}

Se enfoca en las restricciones adicionales de las capacidades limitadas de los vehículos, encargados de distribuir los productos conforme a la demanda de los clientes, considerado como un problema de optimización de tipo NP-Hard (Ochoa-Ortíz et al. 2015).

\section{Programación lineal entera mixta}

El sistema de este modelo de programación puntualiza en la existencia de algunas variables enteras, continuas y binarias (Cornejo Sánchez and Puente 2005). 


\section{Transporte multiproductos}

Implica la traslación de varios productos que se agrupan en las empresas productoras, centradas en ofrecer más de un producto con un gran poder de mercado (Fallis 2013).

\section{Materiales y métodos}

Actualmente, la planificación de estrategias orientadas a los sistemas de distribución de productos en las empresas juega un papel importante en la viabilidad financiera de la misma, debido al margen de contribución con el que esta actividad participa en la estructura de costos de cada uno de estos productos. Se resaltan problemáticas provocadas por la distribución, tales como prioridad de pedidos, vehículo a asignar, condiciones de entrega, producto a entregar y demás variables de alta envergadura (Xing et al. 2017).

\section{Caracterización del sistema de distribución}

La empresa utilizada como objeto de estudio se dedica principalmente a la producción y comercialización de materiales para la construcción, siendo líderes en extracción y aprovechamiento de recursos naturales, los cuales se transforman mediante procesos como trituración y clasificación para ser distribuidos en la región sabana. Nosotros identificamos problemas de planificación en la asignación de rutas y vehículos a pedidos realizados por sus clientes, razón por la cual se procedió a proponer una herramienta matemática que minimizara los costos sujetos a estas decisiones. Para el desarrollo del modelo, se hizo necesario la cuantificación de variables y parámetros inmersos dentro del sistema, entre los cuales se consideraron el modelo conceptual, las demandas de productos y sus especificaciones técnicas. Adicionalmente, se tuvieron en cuenta la flota de vehículos disponibles y sus respectivas capacidades de carga, costos de distribución, cálculo de distancia de nodo a nodo, entre otros.

\section{Modelo conceptual}

El modelo matemático responderá al siguiente gráfico, el cual cuenta con un solo despacho, con $m$ productos y $k$ vehículos, a diferentes nodos $i$, por lo cual la variable $X_{i j k m}$ denotan las cantidades a enviar de cada producto. 
La asignación de la ruta y vehículo se da en la variable $Y_{i j k}$, con el objetivo de maximizar la utilidad.

\section{Portafolio de productos}

Figura 1

Modelo conceptual.

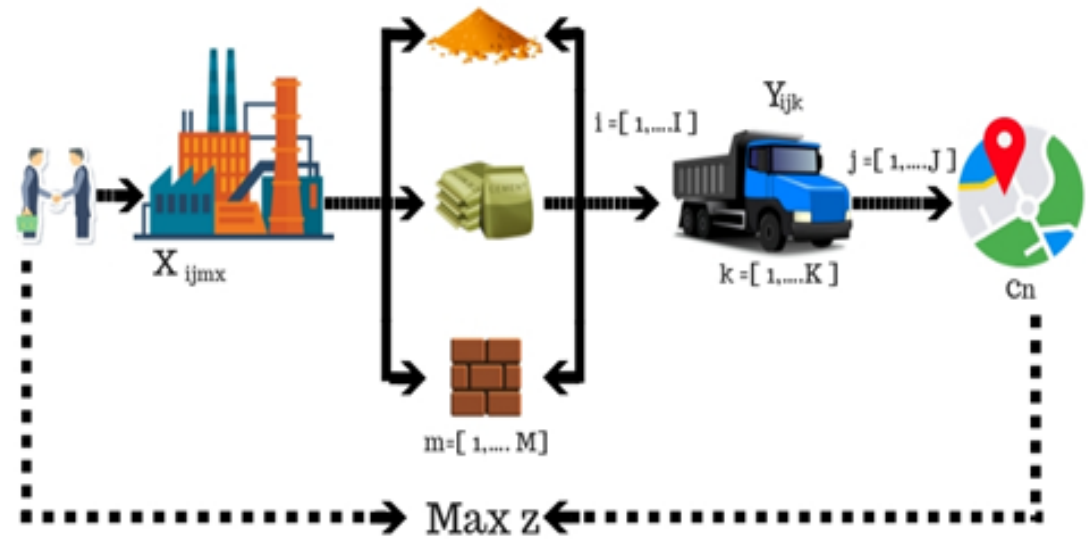

Nota. Fuente: construcción propia.

Esta empresa es capaz de ofrecer una gran variedad de productos para la construcción, en donde es posible identificar diferentes referencias de estos, sin embargo, estas se encuentran inmersas en familias de productos tales como bloques vibrados, calados, gravilla, triturado, polvillo, arena, balastro, sika, cemento, alambre, varilla, Entre otros.

\section{Tabla 1}

Demanda y utilidad semanal de familia de productos.

\begin{tabular}{lcclc}
\hline Producto & Cantidad & Unidad & $\begin{array}{c}\text { Utilidad } \\
\text { unitaria }\end{array}$ & $\begin{array}{c}\text { Utilidad } \\
\text { total }\end{array}$ \\
\hline Gravilla & 132 & $\mathrm{~m}$ & $\$ 50.000$ & $\$ 6.600 .000$ \\
Bloque & 8640 & uds & $\$ 420$ & $\$ 3.628 .800$ \\
Arena & 162 & $\mathrm{~m}$ & $\$ 20.714$ & $\$ 3.355 .668$ \\
Cemento & 600 & $\mathrm{uds}$ & $\$ 3.380$ & $\$ 2.028 .000$ \\
Varillas & 768 & $\mathrm{~m}$ & $\$ 1.500$ & $\$ 1.152 .000$ \\
Balastro & 21 & $\mathrm{Mk}$ & $\$ 18.000$ & $\$ 378.000$ \\
Triturado & 6 & $\mathrm{~m}$ & $\$ 57.000$ & $\$ 342.000$
\end{tabular}




\begin{tabular}{lccll}
\hline Producto & Cantidad & Unidad & $\begin{array}{c}\text { Utilidad } \\
\text { unitaria }\end{array}$ & $\begin{array}{c}\text { Utilidad } \\
\text { total }\end{array}$ \\
\hline Tubo & 24 & $\mathrm{~m}$ & $\$ 8000$ & $\$ 192000$ \\
Sika & 18 & $\mathrm{~kg}$ & $\$ 5000$ & $\$ 90000$ \\
Alambre & 18 & $\mathrm{~kg}$ & $\$ 900$ & $\$ 16200$ \\
Amarre & 18 & Cajas & $\$ 100$ & $\$ 1800$ \\
\hline
\end{tabular}

Nota. Fuente: construcción propia.

Para determinar la importancia de cada una de estas familias en las finanzas de la empresa, se realizó un análisis de Pareto, el cual tuvo como objeto identificar los mayores márgenes de utilidad en ventas por una semana, orientado a cada una de estas familias, logrando mejorar la objetividad del estudio y minimizar los sesgos.

\section{Figura 2}

Diagrama de Pareto enfocado a utilidades semanales por familia de productos.

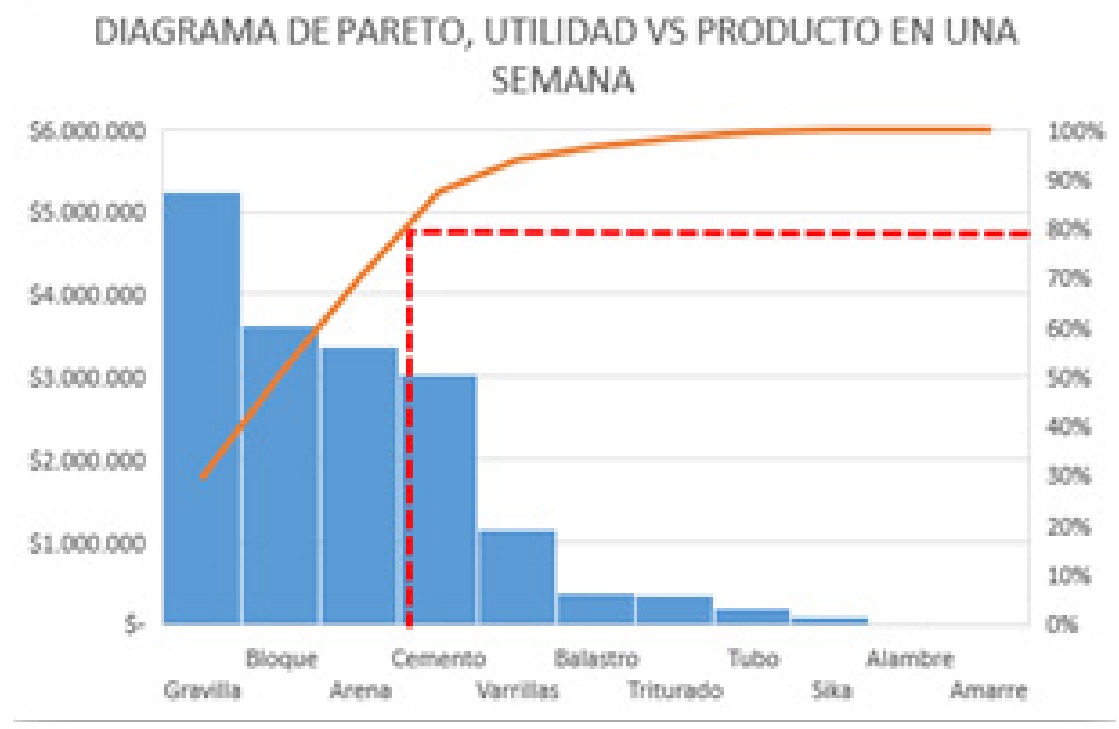

Nota. Fuente: construcción propia.

Ahora bien, a partir del gráfico 1 es posible identificar las familias con mayor contribución semanal en las utilidades de la compañía, entre las cuales se encuentran la gravilla, el bloque, la arena y el cemento, representando el 80\% de las utilidades totales de la empresa en una semana. Cabe resaltar que estas fueron calculadas y seleccionadas para el desarrollo de la investigación, por lo cual se consideran productos. 


\section{Especificaciones técnicas del producto}

Las familias de productos seleccionados presentan deferentes relaciones de peso en toneladas por unidad, dependiendo del tipo de referencia. En este caso, en los productos como la gravilla y la arena se da una relación de 1,2 toneladas por metro cúbico; el bloque, de 0,01 tonelada; y el cemento, de 0,425 toneladas por unidades, respectivamente.

\section{Flota de vehículos}

La empresa cuenta con una flota de vehículos para la distribución de sus productos, entre los cuales se tiene:

Tabla 2

Vehículos para distribución.

\begin{tabular}{|c|c|c|}
\hline \multicolumn{3}{|c|}{ Flota de vehículos } \\
\hline & Camión 300 & Volqueta \\
\hline $\mathrm{N}^{\circ}$ vehículos & 3 & 3 \\
\hline Capacidad (ton) & 5 & 7 \\
\hline
\end{tabular}

Nota. Fuente: construcción propia.

Estos vehículos presentan las siguientes capacidades por cada familia de producto seleccionado

\section{Tabla 3}

Capacidad de cada vehículo por producto.

\begin{tabular}{ccc}
\hline \multicolumn{3}{c}{ Capacidad de cada vehículo por producto en $\mathbf{m}^{\wedge} \mathbf{3}$} \\
\hline Gravilla & Camión 300 & Volqueta \\
Arena & 4,46 & 6,25 \\
Bloque & 4 & 5,6 \\
Cemento & 500 & 700 \\
\hline
\end{tabular}

Nota. Fuente: construcción propia.

Por lo cual se consideran vehículos $k$ y una capacidad de cada vehículo por cada producto . 


\section{Identificación de nodos y cálculo de distancias}

La empresa se encuentra ubicada en el municipio de Sincelejo, en donde posee la mayor concentración de sus clientes, por lo cual tomamos las comunas como base para la asignación de distancias a cada pedido:

Por lo cual se consideran nodos de inicio $i=\{1,2 . ., 9\}$, y la distancia en km del nodo i al nodo $\mathrm{j}\left(\right.$ Dis $\left._{i, j}\right)$.

Figura 3

Grafo de Nodos por comunas.

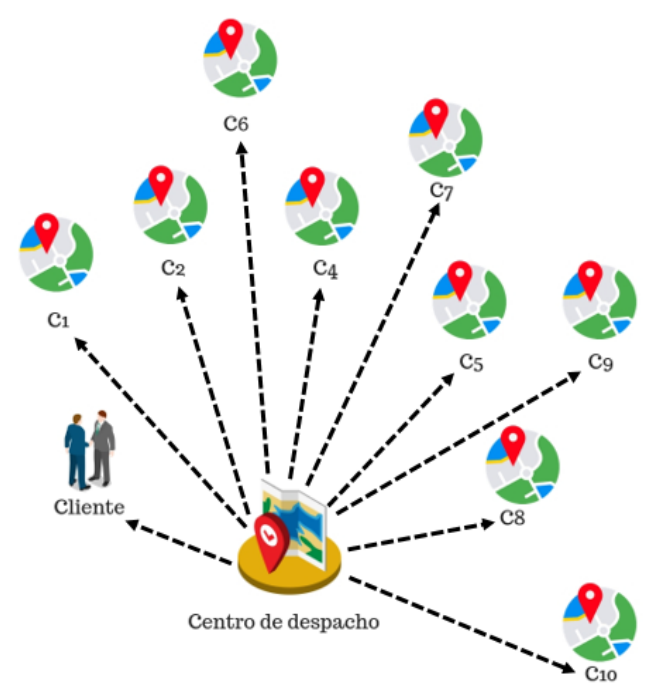

Nota. Fuente: construcción propia.

Figura 4

Mapa del distrito capital de Sucre.

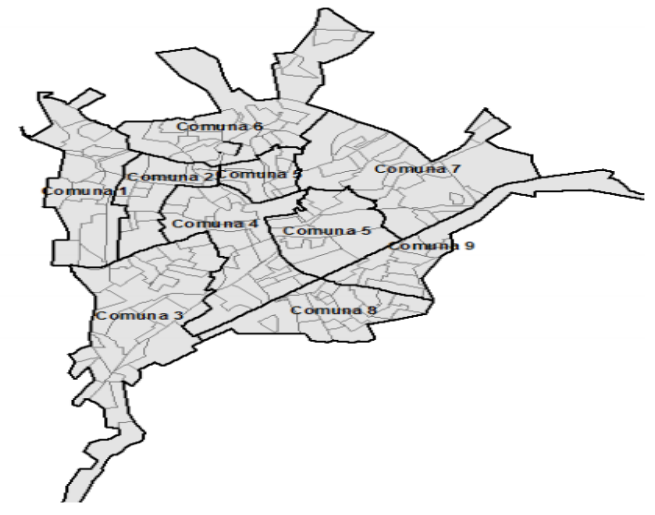

Nota. Fuente: construcción propia. 


\section{Representación gráfica}

La empresa incurre en ciertos costos que se han divido en 3 fases: costos variables, los cuales se deben calcular según los galones de gasolina por kilómetros recorridos; los costos fijos, atribuidos al dinero que se deba cancelar día a día; y los costos asociados a la asignación de cada vehículo a una ruta según su tipo. Los costos se presentan a continuación:

Por lo cual se consideran el costo variable de asignar el vehículo $\mathrm{k}$, por $\mathrm{km}$ recorrido (Cos $t$ Var $_{k}$ ), un costo de asignar el vehículo $\mathrm{k}$ (Cost $A s_{k}$ ) $\mathrm{y}$ un costo fijo de mantener el vehículo $\mathrm{k}$ en la empresa (CostFijo ${ }_{k}$ ), se estipula una base del galón de ACPM en \$ 8.700.

Tabla 4

Costos de distribución del camión 300.

\begin{tabular}{|c|c|c|c|}
\hline \multicolumn{2}{|c|}{ Vehículos } & \multirow{2}{*}{$\begin{array}{c}\text { Combustible } \\
0,14 \mathrm{gl} / \mathrm{km}\end{array}$} & \multirow{2}{*}{$\begin{array}{c}\text { Costos de } \\
\text { viáticos }\end{array}$} \\
\hline Camión & Costo variable & & \\
\hline 300 & Costo de asignar & & 5.000 \\
\hline \multirow{2}{*}{ Volqueta } & Costo variable & $0,103 \mathrm{gl} / \mathrm{km}$ & \\
\hline & Costo de asignar & & 10.000 \\
\hline
\end{tabular}

Nota. Fuente: construcción propia.

\section{Modelo matemático de ruteo capacitado}

El problema de la empresa estudiada se encuentra en el momento de distribuir los productos solicitados por los clientes en sus respectivas localidades, para lo cual, la empresa cuenta con vehículos que realizan dichas actividades. La dificultad radica en cómo programar dichos vehículos para que los recorridos sean los mínimos y puedan disminuir los costos de distribución, para ello, se plantea un modelo de programación lineal mixta de ruteo capacitado (CVRP), dado que son diferentes vehículos con capacidades de distribución heterogéneas.

$$
\begin{aligned}
& \text { Conjuntos } \\
& i=\text { Nodos }, i=\{1,2,3, \ldots, I\} \\
& m=\text { Producto, } m=\{1,2,3, \ldots, M\}
\end{aligned}
$$


$k=$ Vehículos $k=\{1,2,3, \ldots, K\}$

Variables

$X_{i j k m}=$ Cantidad de productos $m$ a enviar

al nodo i,en el vehículo $k$

$Y_{i j k}=1$ si se asigna el vehículo $k$,

con el producto m para el nodo $i$, 0 lo contrario

$Z=$ Utilidad generada por la distribución

$U_{i}=$ Variable de holgura para evitar

sub-ciclos

\section{Parámetros}

$\operatorname{Cap}_{k m}=$ Capacidad de cada vehículo $k$,

con el producto $m$

Ped $_{i m}=$ Pedido a enviar en el i,del producto $m$

CostVar $_{K}=$ Costo variable de asignar el vehículo $k$,

Por kilómetro recorrido

$\operatorname{CostAs}_{K}=$ Costo de asignar el vehículo $k$,

CostFijo = Costo Fijo por la disribución,

Dis $_{i j}=$ Distancia del nodo $i$

$U t i_{m}=$ Utilidad generada por la distribución del

producto $m$

$C G=$ Costo del galón de combustible

$n=$ Cantidad de nodos 


\section{Restricciones}

$$
\begin{gathered}
\operatorname{Max} Z=\sum_{i=1}^{I} \sum_{j=1}^{J} \sum_{k=!}^{K} \sum_{M=1}^{M}\left(X_{i j k m} * U t i_{m}\right) \\
-\sum_{i=!}^{I} \sum_{j=1}^{J} \sum_{k=1}^{K}\left[\left(Y_{i j k} * \operatorname{CostVar}{ }_{k} * \operatorname{Dis}_{i j} * C G\right)+\left(Y_{i j k} * \operatorname{Cost}_{\mathrm{N}}\right)\right] \\
- \text { CostFijo } \\
\sum_{j=1}^{J} \sum_{k=1}^{K} Y_{i j k}=1 \quad \forall i \in N-[1] \\
\sum_{j=1}^{J} Y_{1 j k} \leq 1 \quad \forall k \in N, j-[1]
\end{gathered}
$$

$\sum_{j=1}^{J} \sum_{k=1}^{K} X_{i j m k}-X_{j i m k}=\operatorname{Ped}_{i m} \forall j, m \in N, j-[1] y i \neq j$

$$
\begin{gathered}
X_{i j k m} \leq Y_{i j k} * \operatorname{Cap}_{k m} \forall i, j, k, m \quad \in N, i \neq j \\
\sum_{i=!}^{I} Y_{i, h, k}=\sum_{j=1}^{J} Y_{h, j, k} \quad \forall h, k \in N, i \neq h y j \neq h \\
U_{i}-U_{j}+n * Y_{i j k} \leq n-1 \quad \forall i, j, k \in N, i-[1] y j-[1] \\
X_{i 1 k m}=0 \quad \forall i, j, k, m \\
X_{i 1 k m} \leq M u * Y_{i j k} \forall i, j, k, m \\
\forall X_{i j k m}, U_{i} \geq 0 \\
\forall Y_{i j k m}=[0,1]
\end{gathered}
$$

El anterior modelo declara las variables de cantidad, asignación y holgura del ruteo. Los parámetros descritos y las restricciones actúan de la siguiente forma:

La restricción (1) busca maximizar la utilidad de distribuir los productos; la restricción (2) establece que solo se puede salir una vez por el mismo nodo; la restricción (3) determina que, para una asignación, como 
máximo se puede escoger un vehículo, y no se puede utilizar este para repetir pedidos; la restricción (4) realiza un control de flujo, obligando a que todos los pedidos que realicen los clientes deban cumplirse; la restricción (5) restringe la capacidad de los vehículos; la restricción (6) es una ecuación de balance, con el fin de establecer que el nodo de llegada deba ser igual al nodo de inicio en el siguiente arco del vehículo k, asegurando la consecuencia de la ruta; la restricción (7) controla los subciclos que se puedan generar en las interacciones, logrando igualar la cantidad de nodos existentes con la cantidad de nodos a visitar en el ruteo resultante; la restricción (8) determina que las cantidades al final del ruteo deben ser siempre cero, para todos los productos y vehículos, con el objetivo de que el vehículo siempre regrese a la fábrica sin productos; la restricción (9) realiza un artificio matemático, con el fundamento de que la variable de cantidad, siempre y cuando la binaria lo active, realice alguna actividad; finalmente, las restricciones (10) y (11) determinan la naturaleza de las variables.

\section{Solución}

El modelo fue verificado mediante una captación de pedidos con una ventana de 1 hora, realizando una comparación de lo que la empresa realiza cotidianamente y lo que el modelo arroja.

El modelo planteado fue solucionado con los datos tomados en la empresa, mediante el software GAMS en la versión 23.5, con un procesador AMD A10 - 9620P Radeon R5, demostrando tiempo de solución bajos, logrando obtener el siguiente ruteo. 
Figura 5

Ruteo realizado por la empresa.

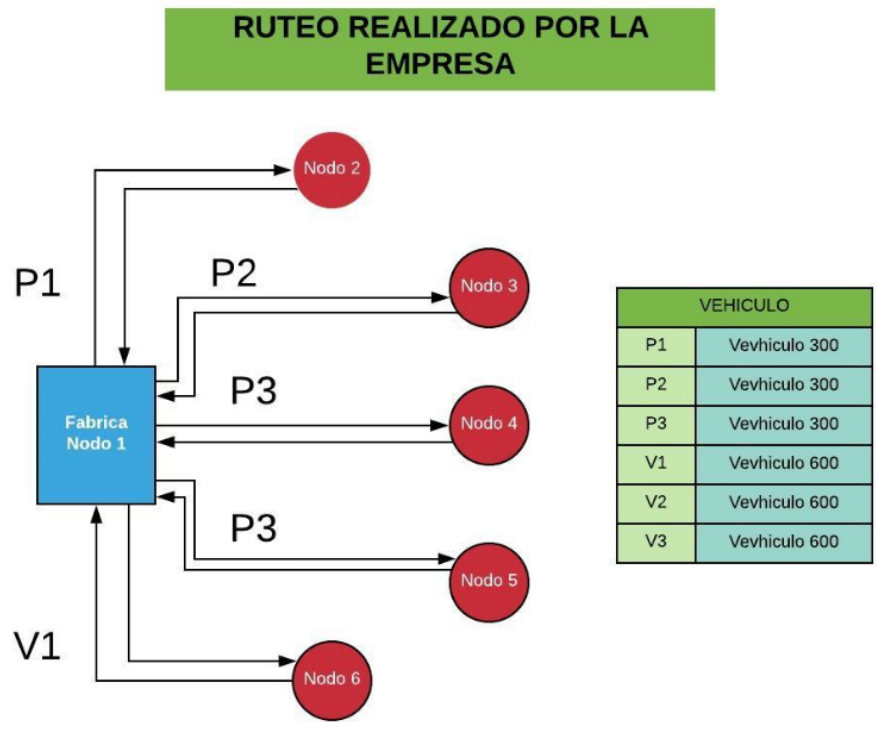

Nota. Fuente: construcción propia.

Tabla 5

Utilidad generada mediante ruteo tradicional.

\section{Utilidad generada mediante ruteo tradicional}

\begin{tabular}{lcccc}
\multicolumn{1}{c}{ Vehículos } & $\begin{array}{c}\text { Costo por } \\
\text { recorrido }\end{array}$ & $\begin{array}{c}\text { Costo por } \\
\text { asignación }\end{array}$ & $\begin{array}{c}\text { Costos } \\
\text { fijos }\end{array}$ & Beneficio \\
\hline Camión 300-1 & $\$ 9.013$ & $\$ 10.000$ & & $\$ 180.080$ \\
Camión 300-2 & $\$ 4.385$ & $\$ 10.000$ & $\$$ & $\$ 70.714$ \\
Camión 300-3 & $\$ 12.911$ & $\$ 10.000$ & 15.000 & $\$ 54.514$ \\
Volqueta - 1 & $\$ 5.568$ & $\$ 30.000$ & & $\$ 83.428$ \\
Total & & $\$ 106.877$ & & $\$ 388.736$ \\
Utilidad & & & & $\$ 281.859$ \\
\hline
\end{tabular}

Nota. Fuente: construcción propia. 
Modelo de ruteo de vehículos capacitado y multiproducto aplicado a una empresa distribuidora de materiales para la construcción en Sincelejo, Sucre

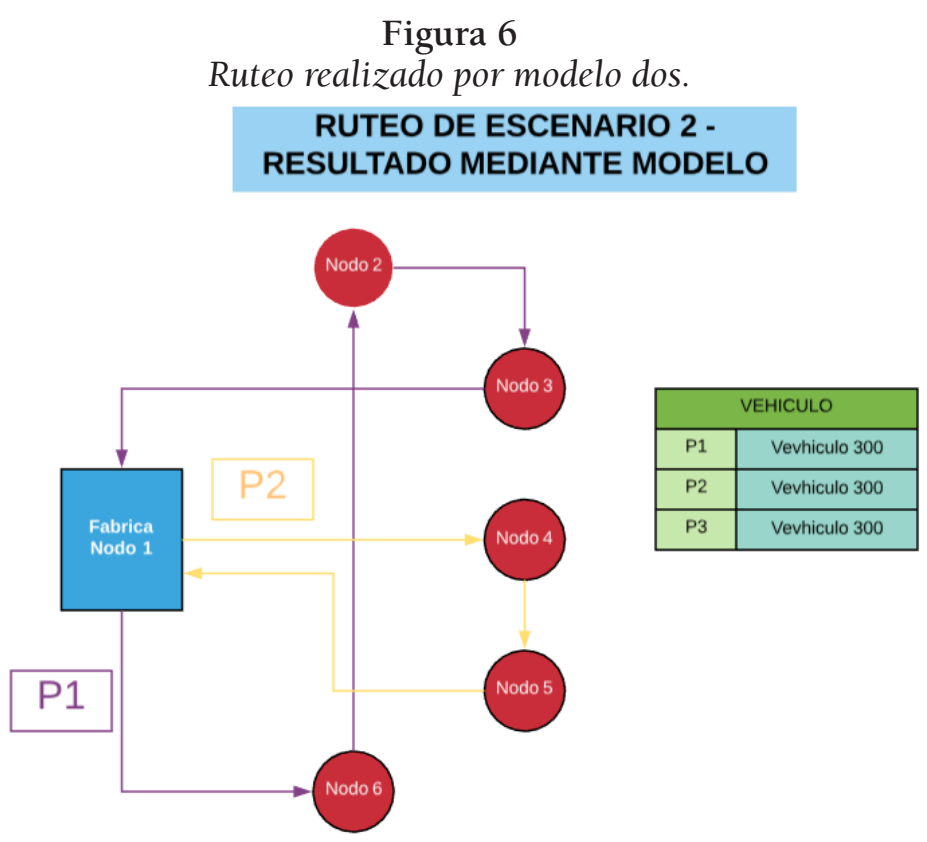

Nota. Fuente: construcción propia.

Es necesario un cambio de ruta dado que la empresa envía los pedidos cada que esta se ejecuta, provocando desperdicios de capacidad en ciertos vehículos. 


\title{
Resultado y discusión
}

\author{
Figura 7
}

Ruteo realizado por el modelo tres.

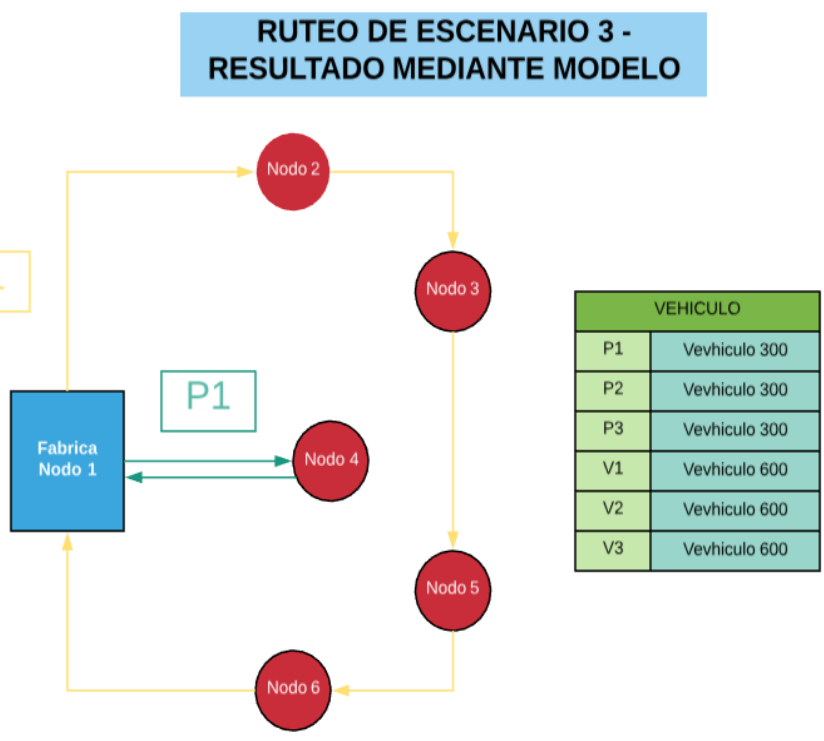

Nota. Fuente: construcción propia.

El ruteo realizado por la empresa dispone de 4 camiones, debe entregar y volver a cargar el pedido, creando excesos de tiempo de entrega.

El ruteo realizado por el modelo utiliza 2 vehículos, aprovechando al máximo las capacidades de cada vehículo, aumentando el beneficio por distribuir los productos.

Tabla 7

Utilidad obtenida mediante el modelo.

\begin{tabular}{ccccc}
\hline \multicolumn{4}{c}{ Utilidad generada mediante modelo matemático } & \\
Vehículos & $\begin{array}{c}\text { Costo por } \\
\text { recorrido }\end{array}$ & $\begin{array}{c}\text { Costo por } \\
\text { asignación }\end{array}$ & Costos fijos & Beneficio \\
\hline Camión & $\$ 6.090$ & $\$ 10.000$ & $\$ 15.000$ & $\$ 33.800$ \\
$300-1$ & $\$ 22.620$ & $\$ 30.000$ & $\$ 354.936$ & \\
Volqueta-1 & & $\$ 83.710$ & \\
Total & & $\$ 305.026$ & $\$ 388.736$ \\
Utilidad & & &
\end{tabular}




\begin{tabular}{|c|c|c|c|c|}
\hline \multicolumn{5}{|c|}{ Utilidad generada mediante modelo matemático } \\
\hline Vehículos & $\begin{array}{l}\text { Costo por } \\
\text { recorrido }\end{array}$ & $\begin{array}{l}\text { Costo por } \\
\text { asignación }\end{array}$ & Costos fijos & Beneficio \\
\hline \multicolumn{5}{|c|}{ Utilidades } \\
\hline \multicolumn{3}{|c|}{ Ruteo tradicional } & \multicolumn{2}{|r|}{$\$ 281.859$} \\
\hline \multicolumn{3}{|c|}{ Ruteo del modelo escenario dos } & \multicolumn{2}{|r|}{$\$ 282.508$} \\
\hline \multicolumn{3}{|c|}{ Ruteo del modelo escenario tres } & \multicolumn{2}{|r|}{$\$ 305.026$} \\
\hline
\end{tabular}

Nota. Fuente: construcción propia.

El ruteo de la empresa generó un beneficio de \$281.859; el presentado por el modelo en el escenario 2, \$282.508; y en el escenario 3, \$ 305.206, produciendo un incremento de $0.23 \%$ y $8.22 \%$, respectivamente, para los dos últimos casos. Esto es ocasionado en un solo ruteo de los 10 o 15 que la organización realiza a diario.

\section{Conclusiones}

El problema de ruteo capacitado (CVRP), modelado matemáticamente mediante programación lineal mixta, obtiene resultados muy favorables para las organizaciones, representando mejoras en el ruteo con tiempos de solución bajos, ayudando al área de despacho a tomar decisiones que maximicen el beneficio de la empresa.

La empresa actualmente asigna los vehículos en el momento de generación del pedido, con la intención de disminuir los tiempos de entrega, aunque esto provoca desperdicios de capacidad y obliga a fijar mayor cantidad de vehículos para los mismos pedidos.

Esta investigación contribuye a las nuevas propuestas de ruteo de vehículos, determinando un modelo matemático multiproductos, con diferente capacidad, acercándose un poco más a las necesidades reales de las empresas.

\section{Agradecimientos}

A Dios por bendecirnos, por guiarnos en nuestra existencia, ser el apoyo y fortaleza en nuestra carrera. 
A la empresa referenciada en el estudio de nuestra investigación, por la oportunidad y el apoyo brindado.

Al Ing. José Ruiz Meza que con su sabiduría y conocimiento fue nuestra guía en el desarrollo de esta investigación.

\section{Referencias}

Bermeo Muñoz, Elver A., and Jaime Hernán Calderón Sotero. 2009. "Diseño de Un Modelo de Optimización de Rutas de Transporte." El Hombre y La Máquina 32(enero-junio):52-67.

Cornejo Sánchez, Christian, and Miguel Mejía Puente. 2005. "Modelo de Programación Lineal Entera Mixta Para El Planeamiento de Las Importaciones En Régimen Aduanero Definitivo PARTE I The Mixed Integer Linear Programming Model for the Import Planning in Definitive Customs Regime PART I.'

Fallis, A. .. 2013. "Análisis De Las Características Y Aplicaciones De Los Sistemas De Ruteo De Vehículos." Journal of Chemical Information and Modeling 53(9):1689-99. doi: 10.1017/CBO9781107415324.004.

González Vargas, Guillermo, and Felipe González Aristizábal. 2006. "Metaheurísticas Aplicadas Al Ruteo de Vehículos. Un Caso de Estudio. Parte 1: Formulación Del Problema." Revista Ingeniería e Investigación 26(3):149-57.

Integrado, Modelo, De Capacidad De Madurez, Hugo Arboleda, Andrés Paz, and Rubby Casallas. 2013. "Estudios Gerenciales." 29(122):17788. doi: 10.1016/j.estger.2014.09.003.

Lama, Miranda-de, and C. Genaro. 2013. "Artículo de Revisión Transporte y Logística Pre-Sacrificio :" 44:31-56.

Ochoa-Ortíz, Alberto, Francisco Ornelas-Zapata, Lourdes Margain-Fuentes, Miguel Gastón Cedillo-Campos, Jöns Sánchez-Aguilar, Rubén Jaramillo-Vacio, and Isabel Ávila. 2015. "Capacitated Vehicle Routing Problem for PSS Uses Based on Ubiquitous Computing: An Emerging Markets Approach." Dyna 82(191):20-26. doi: 10.15446/ dyna.v82n191.51141.

Tokito, Shohei. 2018. "Environmentally-Targeted Sectors and Linkages in the Global Supply-Chain Complexity of Transport Equipment." 
Modelo de ruteo de vehículos capacitado y multiproducto aplicado a una empresa distribuidora de materiales para la construcción en Sincelejo, Sucre

Ecological Economics 150(July 2017):177-83. doi: 10.1016/j.ecolecon.2018.04.017.

Xing, Meiyu, Kwame Awuah-Offei, Suzanna Long, and Shoaib Usman. 2017. "The Effect of Local Supply Chain on Regional Economic Impacts of Mining." Extractive Industries and Society 4(3):622-29. doi: 10.1016/j.exis.2017.05.005. 